\title{
Microbiota, type 2 diabetes and non-alcoholic fatty liver disease: protocol of an observational study
}

\author{
Benedetta M. Motta ${ }^{1 *+} \odot$, Christoph Grander ${ }^{2 \dagger}$, Martin Gögele ${ }^{1}$, Luisa Foco ${ }^{1}$, Vladimir Vukovic', \\ Roberto Melotti ${ }^{1}$, Christian Fuchsberger ${ }^{1}$, Alessandro De Grandi ${ }^{1}$, Chiara Cantaloni ${ }^{1}$, Anne Picard ${ }^{1}$, \\ Deborah Mascalzoni ${ }^{1,3}$, Alessandra Rossini ${ }^{1}$, Cristian Pattaro ${ }^{1}$, Herbert Tilg $^{2^{*+}}$ and Peter P. Pramstaller ${ }^{1 \dagger}$
}

\begin{abstract}
Background: Non-alcoholic fatty liver disease (NAFLD) is characterized by triglyceride accumulation in the hepatocytes in the absence of alcohol overconsumption, commonly associated with insulin resistance and obesity. Both NAFLD and type 2 diabetes (T2D) are characterized by an altered microbiota composition, however the role of the microbiota in NAFLD and T2D is not well understood. To assess the relationship between alteration in the microbiota and NAFLD while dissecting the role of T2D, we established a nested study on T2D and non-T2D individuals within the Cooperative Health Research In South Tyrol (CHRIS) study, called the CHRIS-NAFLD study. Here, we present the study protocol along with baseline and follow-up characteristics of study participants.
\end{abstract}

Methods: Among the first 4979 CHRIS study participants, 227 individuals with T2D were identified and recalled, along with 227 age- and sex-matched non-T2D individuals. Participants underwent ultrasound and transient elastography examination to evaluate the presence of hepatic steatosis and liver stiffness. Additionally, sampling of saliva and faeces, biochemical measurements and clinical interviews were carried out.

Results: We recruited 173 T2D and 183 non-T2D participants (78\% overall response rate). Hepatic steatosis was more common in T2D (63.7\%) than non-T2D (36.3\%) participants. T2D participants also had higher levels of liver stiffness (median $4.8 \mathrm{kPa}$, interquartile range (IQR) 3.7, 5.9) than non-T2D participants (median $3.9 \mathrm{kPa}$, IQR 3.3, 5.1). The noninvasive scoring systems like the NAFLD fibrosis score (NFS) suggests an increased liver fibrosis in T2D (mean -0.55 , standard deviation, SD, 1.30) than non-T2D participants (mean - 1.30, SD, 1.17).

Discussion: Given the comprehensive biochemical and clinical characterization of study participants, once the bioinformatics classification of the microbiota will be completed, the CHRIS-NAFLD study will become a useful resource to further our understanding of the relationship between microbiota, T2D and NAFLD.

Keywords: Microbiota, Type 2 diabetes, NAFLD, Cooperative Health Research In South Tyrol

\footnotetext{
*Correspondence: benedetta.motta@eurac.edu; herbert.tilg@i-med.ac.at

${ }^{\dagger}$ Benedetta M. Motta and Christoph Grander contributed equally to this

work

${ }^{\dagger}$ Herbert Tilg and Peter P. Pramstaller contributed equally to this work

${ }^{1}$ Institute for Biomedicine (affiliated to the University of Lübeck), Eurac

Research, 39100 Bolzano, Italy

2 Department of Internal Medicine I, Gastroenterology, Hepatology,

Endocrinology \& Metabolism, Medical University Innsbruck, Innsbruck,

Austria

Full list of author information is available at the end of the article
}

(c) The Author(s) 2019. This article is distributed under the terms of the Creative Commons Attribution 4.0 International License (http://creativecommons.org/licenses/by/4.0/), which permits unrestricted use, distribution, and reproduction in any medium, provided you give appropriate credit to the original author(s) and the source, provide a link to the Creative Commons license, and indicate if changes were made. The Creative Commons Public Domain Dedication waiver (http://creativecommons.org/ publicdomain/zero/1.0/) applies to the data made available in this article, unless otherwise stated. 


\section{Background}

Non-alcoholic fatty liver disease (NAFLD) encompasses a spectrum of disorders characterized by hepatic triglyceride accumulation (hepatic steatosis) in the absence of alcohol overconsumption [1]. Twenty-to-thirty \% of the NAFLD patients progress to non-alcoholic steatohepatitis (NASH), implying liver inflammation and association with liver-related diseases such as fibrosis, cirrhosis, and hepatocellular carcinoma [2]. NAFLD has an estimated prevalence of about $25 \%$ in the general population [3] and is expected to become the leading cause of liver transplantation over the next 20 years, with expanding costs for healthcare systems [4]. In the presence of type 2 diabetes (T2D) and metabolic syndrome (MetS), NAFLD prevalence can rise up to 70\% [5-7]. T2D may increase the risk of developing NAFLD and NASH, but also NAFLD itself may be a risk factor for T2D onset $[8,9]$. The interplay between NAFLD, T2D, and MetS is complex, with NAFLD and MetS sharing clinical manifestations such as obesity, insulin resistance, T2D, dyslipidemia, and hypertension [10].

Triggering factors, such as the translocation of bacterial components and their products from the gut into the systemic circulation following alterations of the intestinal integrity, have been identified as an important mechanism of NAFLD onset [11, 12]. Animal studies suggest that bacterial components may also have a crucial role in NAFLD and NASH onset in humans [13].

The gut microbiota represents the community of microorganisms inhabiting the digestive tract, with vital functions in relation to vitamin biosynthesis, bile acid degradation, maintenance of the intestinal mucosal barrier integrity, and complex carbohydrate digestion. Microbiota composition can be altered by medications, environmental factors, and diet [14, 15]. Individuals with T2D [16-18] or MetS [19] may suffer a disrupted intestinal microbial composition, which then promotes an imbalance between protective and harmful effects of the microbiota on the host. Increasing evidence suggests an important role for the intestinal microbiota in the pathogenesis of T2D, regulating metabolic pathways and glucose hemostasis [20]. For example, obesity is associated with a larger number of bacterial strains that ferment food components and increase the potential of the host to harvest energy [21]. Obese and MetS-affected individuals may have an altered ratio of Firmicutes-toBacteroidetes specific phyla [22, 23]. Members of both phyla are involved in the production of short chain fatty acids (SCFAs) from dietary compounds not completely digested in the small intestine [24]. Evidence suggests a role of SCFAs as key mediators of the cross talk between brain and gut in the pathogenesis of obesity [25]. It has been shown that the total amount of SCFA produced is higher in obese subjects, suggesting that SCFA metabolism might play a considerable role in obesity [26]. Recently, using bidirectional Mendelian randomization, the causal relationship between the gut microbiome and metabolic traits has been explored, providing evidence of a causal effect of the gut microbiome on metabolic traits [27]. Several studies have shown alterations of the microbiota composition in NAFLD [28-30] and NASH [31] patients. Increased relative abundance of Bacteroides and Ruminococcus in the intestine has been associated with more severe histology in NAFLD patients [32]. In contrast, NAFLD patients seem to have lower relative abundance of the Prevotella strain [32, 33]. Recently, 37 bacterial strains from the gut were identified that allowed discrimination between mild and severe hepatic fibrosis in biopsy-proven NAFLD patients [34].

Another important microbial habitat is the oral cavity [35]. In addition to contributing to oral diseases [36, 37], the oral microbiota may represent a risk factor for systemic diseases such as T2D [38, 39]. NAFLD was associated with periodontitis, and some characteristics of periodontitis such as systemic inflammation and invasion of the commensal bacteria are involved in progression of liver fibrosis in NAFLD-affected individuals [40]. Experimental models showed an association between Porphyromonas gingivalis and risk of NAFLD and NASH [41, 42]. The importance of microbial invasion from the oral cavity into the lower intestine compartments in patients with cirrhotic liver disease was recently demonstrated by a study showing that $>50 \%$ of bacterial species found to be enriched in the intestine of cirrhotic patients were of buccal origin [43]. While the oral microbiota has gained much attention only recently, there remain many unanswered questions on the role of bacterial strains on specific pathologies of the liver.

To further improve the general understanding on the relationship between the microbiota, from gut and mouth, and NALFD, and to illuminate such relationships in the context of T2D, we carried out an observational study nested within the Cooperative Health Research in South Tyrol (CHRIS) study [44], called CHRIS-NAFLD. Here, we describe the study protocol, recruitment and measurement procedures, and offer a description of the epidemiological characteristics of study participants at the baseline and follow-up times.

\section{Methods \\ Study design}

The CHRIS-NAFLD study was setup in the context of the CHRIS study, a population-based study carried out in a rural Alpine context [44, 45]. CHRIS study participants were on overnight fasting, underwent blood and urine collection, anthropometric and blood pressure (BP) 
measurements, electrocardiographic analysis, and tremor assessment. Participants were also administered a series of interviewer- and self-administered questionnaires about their health status. The CHRIS-NAFLD study was designed after the recruitment of the first 4979 CHRIS study participants, which was carried out between 2011 and 2014 (baseline data) [44]. From the 4979 participants, we selected for the CHRIS-NAFLD study all 227 individuals affected by T2D and an equal number of non-T2D individuals, matched on age ( \pm 2 years tolerance) and sex. T2D was defined according to standard guidelines [46], as a positive response to the question "Has a doctor ever diagnosed you with diabetes?" or either having fasting plasma glucose levels of $\geq 126 \mathrm{mg} / \mathrm{dl}$ or glycated hemoglobin (HbA1c) levels of $\geq 6.5 \%$. Participants with other types of diabetes were excluded from the selection. Among participants selected as non-T2D diabetes (negative response to the doctor's diagnosed diabetes question), participants were further excluded in case of HbA1c levels beyond 5.6\%, to prevent inclusion of potentially pre-diabetic participants.

\section{Recruitment of study participants}

Selected participants were informed about the objective and content of the CHRIS-NAFLD study by invitation letter, mailed between 2 and 3 weeks prior to enrollment. They were subsequently contacted by phone to arrange an appointment at the study center. The participants were requested not to eat anything from 8:00 pm of the night before the day of participation, and to abstain from drinking or smoking for at least $2 \mathrm{~h}$ prior to the visit. At the study center, participants underwent blood drawing, urine collection, saliva sampling, anthropometric and BP measurements, tremor assessment, and clinical examination by a medical doctor to assess the hepatic status. Body mass index (BMI), fat percentage, and visceral and subcutaneous fat were assessed using a body composition monitor (OMRON BF508). Waist and hip circumference were measured according to the WHO protocol [47].

\section{Questionnaires and interview}

Questionnaires concerning the change of participants' health status since the baseline participation and their life-style were administered by an interviewer, and the medication intake in the last 7 days was documented using an electronic optical scan of their medication box bar codes according to the Anatomical Therapeutic Chemical (ATC) classification system. A Food Frequency Questionnaire (FFQ) based on the Global Allergy and Asthma European Network of Excellence study [48] was mailed to their homes prior to participation, in order to limit the time spent at the study center. The FFQ also asked about the average frequency of the consumption of alcoholic drinks during the last 12 months (rarely or never, 1-3/month, 1/week, 2-4/week, 5-6/week, 1/day, $2+$ /day), specifically of beer $(200 \mathrm{ml})$, red wine $(125 \mathrm{ml})$, white wine $(125 \mathrm{ml})$, rosé wine $(125 \mathrm{ml})$, liqueurs $(50 \mathrm{ml})$, and spirits $(50 \mathrm{ml})$. At the study center, participants answered a computer-assisted interviewer-administered questionnaire on smoking habits, based on the European Community Respiratory Health Survey II [49] from which we derived pack-years as a measure of cumulative smoking. Based on their smoking habits, participants were classified as never smokers (never smoked or smoked for $<1$ year in their lifetime), past-smokers (smoked for $\geq 1$ year in their lifetime but stop smoking $\geq 1$ year before the interview), and current-smokers (currently smoking at the time of the interview or stopped smoking $<1$ month before the interview).

\section{Evaluation of the hepatic steatosis and fibrosis}

To evaluate the presence of hepatic steatosis and fibrosis, participants underwent abdominal ultrasound examination (5-1 MHz Phased Array Transducer, iViz, SonoSite, USA) and transient elastography (Fibroscan ${ }^{\circledR}$, Echosens, France) performed by a trained medical doctor (Christoph Grander).

To assess transient elastography (TE), participants were placed in a supine position with their right arm fully adducted and asked to hold their breath. At least ten independent resistance measurements were taken, starting always with an $\mathrm{M}+$ probe but using an $\mathrm{XL}+$ probe as a backup option when prompted by the automatic probe selection tool $[50,51]$. TE values were defined as unreliable when the IQR to median ratio was $>30 \%$. Fibrosis was then scaled into four stages, F0 to F4, based on the resistance levels of the liver [52]. TE values of $>6.5 \mathrm{kPa}$ were considered as diagnosis of fibrosis ( $\geq$ F1) [53].

Additionally, abdominal ultrasound scanning was performed in every participant after overnight fasting. Presence and severity of steatosis was evaluated as documented by Ballestri et al. [54]. Steatosis was classified into three grades: normal or very slight increase in the echo pattern with normal visualization of vessels and diaphragm (grade 1); moderate increase in echogenicity with reduced visibility of portal veins and diaphragm (grade 2 ); or distinct increase in echo pattern with poor visibility of intrahepatic vessels and diaphragm (grade 3). Presence of gallstones, gallbladder size, and wall thickness were also assessed, as well as the visceral and subcutaneous fat thickness to gain insights into the participant's metabolic risk profile [55].

The presence of NAFLD was defined as steatosis grade of $\geq 2$, after the exclusion of other causes such as overt hepatitis due to virus infection, or hereditary liver disorders or other liver diseases [56]. Three participants were 
excluded from further analyses because of potential drug induced steatosis by methotrexate. Participants were not excluded based on alcohol consumption levels.

Advanced fibrosis was defined as LSM values $\geq 6.5 \mathrm{kPa}$

For a deeper characterization of steatosis and fibrosis, additional surrogate markers have been calculated as reported in Box 1.

\section{Box 1. Evaluation of MetS and hepatic steatosis/fibrosis}

Metabolic syndrome (MetS) $[57,58]$. Presence of 3 risk factors:

Must have:

Central obesity (WC $\geq 94 \mathrm{~cm}$ in males and $\geq 80 \mathrm{~cm}$ in females).

Plus any two of the following four factors:

- TG level: $\geq 150 \mathrm{mg} / \mathrm{dl}(1.7 \mathrm{mmol} / \mathrm{l})$, or specific treatment for this lipid abnormality.

- HDL cholesterol: $<40 \mathrm{mg} / \mathrm{dl}(1.03 \mathrm{mmol} / \mathrm{l})$ in males and $<50 \mathrm{mg} / \mathrm{dl}(1.29 \mathrm{mmol} / \mathrm{l})$ in females, or specific treatment for this lipid abnormality.

- Systolic BP $\geq 130$ or diastolic BP $\geq 85 \mathrm{~mm} \mathrm{Hg}$, or treatment of previously diagnosed hypertension.

- $\mathrm{FPG} \geq 100 \mathrm{mg} / \mathrm{dl}$ ( $5.6 \mathrm{mmol} / \mathrm{l})$, or previously diagnosed T2D.

Visceral adiposity index (VAI) [59]

Males: (WC $\left.[\mathrm{cm}] / 39.68+1.88 \cdot \mathrm{BMI}\left[\mathrm{kg} / \mathrm{m}^{2}\right]\right) \cdot \mathrm{TG}$

$[\mathrm{mmol} / \mathrm{l}] / 1.03 \cdot 1.31 / \mathrm{HDL}[\mathrm{mmol} / \mathrm{l}]$

Females: (WC $\left.[\mathrm{cm}] / 36.58+1.89 \cdot \mathrm{BMI}\left[\mathrm{kg} / \mathrm{m}^{2}\right]\right) \cdot \mathrm{TG}$

$[\mathrm{mmol} / \mathrm{l}] / 0.81 \cdot 1.52 / \mathrm{HDL}[\mathrm{mmol} / \mathrm{l}]$

NAFLD liver fat score (LFS) [60]

NAFLD-LFS: $-2.89+1.18 \cdot$ MetS $[$ yes $=1$, no $=0]+$ $0.90 \cdot$ T2D $[$ yes $=1$, no $=0]+0.15 \cdot$ insulin $[\mathrm{mU} / \mathrm{l}]+$ $0.04 \cdot$ AST $[\mathrm{U} / \mathrm{l}]-0.94 \cdot \mathrm{AST}[\mathrm{U} / \mathrm{l}] / \mathrm{ALT}[\mathrm{U} / \mathrm{l}]$

Hepatic steatosis score (HSI) [61]

HSI: 8 - ALT $[\mathrm{IU} / \mathrm{l}] / \mathrm{AST}[\mathrm{IU} / \mathrm{l}]+\mathrm{BMI}\left[\mathrm{kg} / \mathrm{m}^{2}\right](+2$ if $\mathrm{T} 2 \mathrm{D} ;+2$ if female)

Fatty liver index (FLI) [62]

FLI $=\mathrm{e}^{\theta} /\left(1+\mathrm{e}^{\theta}\right) \cdot 100$, where $\theta=0.953 \cdot \ln (\mathrm{TG}$ $[\mathrm{mmol} / \mathrm{l}])+0.139 \cdot \mathrm{BMI}\left[\mathrm{kg} / \mathrm{m}^{2}\right]+0.718 \cdot \ln (\mathrm{GGT}$ $[\mathrm{U} / \mathrm{l}])+0.053 \cdot \mathrm{WC}[\mathrm{cm}]-15.745$

NAFLD fibrosis score (NFS) [63]
NFS: $-1.675+0.037 \cdot$ age [years] $+0.094 \cdot$ BMI $[\mathrm{kg} /$ $\left.\mathrm{m}^{2}\right]+1.13 \cdot$ IFG or DM [yes $=1$, no $\left.=0\right]+0.99$. AST/ALT $-0.013 \cdot$ PLT $\left[\times 10^{-9} / 1\right]-0.66 \cdot$ albumin $[\mathrm{g} / \mathrm{dl}]$

Fibrosis-4 (FIB-4) [64]

Fib-4: (age [years] · AST [U/l]) / (PLT $\left[10^{9} / \mathrm{l}\right] \cdot$ ALT $\left.[\mathrm{U} / \mathrm{l}]^{1 / 2}\right)$

Homeostatic model assessment-insulin resistance (HOMA-IR) [65]

HOMA-IR: FPG [mg/dl] • insulin [mU/l]/405

\section{Biospecimen collection and biobanking}

For the CHRIS-NAFLD study, blood $(49 \mathrm{ml})$ and urine $(30 \mathrm{ml})$ samples for laboratory analysis and biobanking were collected in the early morning, after an overnight fasting, following the same procedures previously described for the CHRIS study in terms of sample preanalytical processing, transportation, and biobanking [44, 45]. In addition, two $1 \mathrm{ml}$ aliquots of serum were stored at $-80^{\circ} \mathrm{C}$ and sent in dry ice in a unique batch at the end of the recruitment to Synlab Italia Srl for insulin measurement. The CHRIS biobank was assigned a "Bioresource Research Impact Factor" code BRIF6107 [44, 66].

\section{Stool and saliva collection for the metagenomic analysis}

Stool collection tubes were shipped to the participant's home some days before the enrollment with instructions for the sample collection. Participants were asked to collect the samples in the same morning of their visit or, failing that, within $24 \mathrm{~h}$ of the visit. Participants brought their stool samples to the study center at room temperature. Once at the study center, samples were stored at $-20{ }^{\circ} \mathrm{C}$. Samples were then transported frozen to the biobank, where they were finally stored at $-80^{\circ} \mathrm{C}$. Information about the exact time of defecation was collected and the Bristol stool scale (BSS) was assessed [67]. The BSS is used to classify stool consistence: it can be used as a surrogate marker for stool transit time [68] and is applied in both clinical and experimental fields [69].

Non-stimulated saliva samples were collected using the Omnigene oral collection device (OM-501, DNA Genotek, USA) at the study center. After collection, stabilized saliva samples were transported to the biobank, where they were stored at $-80^{\circ} \mathrm{C}$ after splitting into $500 \mu \mathrm{l}$ aliquots. Status of teeth and gums was assessed using items 3,6 , and 12 of the WHO's Oral Health Questionnaire (OHQ) for Adults [70]. 


\section{Microbiome extraction protocol}

DNA extraction from the faeces was performed using a Chemagic Magnetic Separation Module I Dispenser (Perkin Elmer, USA) according to the Chemagen protocol (Chemagic DNA Feces $1 \mathrm{k}$ drying prefilling H12 VD160617.che) using a blood kit (CMG-763-1, Perkin Elmer) supplemented with a lysis buffer specifically for stools (CMG-852, Perkin Elmer). Briefly, under a sterile hood, up to $1 \mathrm{~g}$ of each sample was taken and immediately immersed in $8 \mathrm{ml}$ lysis buffer. The weight was annotated and the tube mixed thoroughly on a vortex. After adding $50 \mu \mathrm{l}$ protease mix (provided in the kit), the sample was incubated for $20 \mathrm{~min}$ at $70{ }^{\circ} \mathrm{C}$ followed by $5 \mathrm{~min}$ inactivation at $95^{\circ} \mathrm{C}$. The sample was centrifuged and the supernatant transferred to a new tube that was further processed on the robot.

For the DNA extraction protocol from saliva, based on chemical lysis and purification for downstream applications, we followed the manufacturer's protocol (CMG1037, Chemagic DNA Saliva Kit special, Perkin Elmer). DNA was quantified with QuantiFluor (E2670, Promega) on an Envision plate reader (Perkin Elmer) and quality was assayed on a NanoDrop spectrophotometer (ThermoFisher, USA) and by running on a $0.5 \%$ agarose gel.

Metagenomic sequencing will be based on the amplification of the V3-V4 hypervariable region of the $16 \mathrm{~S}$ RNA gene with specific primers which can then be used to incorporate unique indexes into the fragments which will further allow unique indexing of up to 384 samples that can then be pooled together. The resulting multiplexed pool will be run on the MiSeq System using the V3 chemistry 600 cycle kit (16S Metagenomic Sequencing Library Preparation System, Illumina, USA).

\section{$16 \mathrm{~S}$ data processing and analysis}

We will follow the data processing and the analytical pipeline developed by the MiBioGen consortium [71], comprising the following steps: $16 \mathrm{~S}$ data processing, genotype data processing, and genome-wide association study (GWAS) [71]. For the $16 \mathrm{~S}$ data processing, we will use the Ribosomal Database Project (RDP) Classifier instead of OTU picking, since it leads to more consistent results and for the genotype imputation we will use the freely available Michigan Imputation Server [72]. For the GWAS analysis we will follow the uniform analytical pipeline developed by the consortium.

\section{Statistical analyses and power calculation}

The association between microbiota composition and NAFLD will be assessed using the Fisher's exact test for proportions. We built power scenarios using the "power two-proportions" command with the "test(fisher)" option implemented in Stata version 14. The impact of predictor variables on the presence of NAFLD or NAFLD-related symptoms and markers will be determined using univariable and multivariable logistic regression models. The role of T2D in the microbiome-NAFLD relationship will be investigated by means of interaction analyses. To assess the relationship between NAFLD severity and microbiota we will fit linear regression models. All models will be further controlled for participants' relatedness to avoid biased estimates due to population structure. Finally, we will analyze the difference between microbiota measured from saliva and from stools in participants with NAFLD and in those with T2D.

\section{Ethical considerations}

The CHRIS-NAFLD study protocol was approved by the Ethical Committee of the Healthcare System of the Autonomous Province of Bolzano (Südtiroler Sanitätsbetrieb/Azienda Sanitaria dell'Alto Adige), protocol no. 85-2016 (19 Oct 2016). As it is nested within the CHRIS study, the CHRIS-NAFLD study follows the CHRIS's ethics protocols with regard to the collection, use and access of data and biosamples, which are stored for a long-term (30 years) after the end of recruitment. Participants were recruited based on a previous consent that allowed recall. The CHRIS study uses online dynamic consent procedures for empowering the autonomy and compliance of study participants. The dynamic consent options for re-contact, allowed us to re-invite the participants for the CHRIS-NAFLD study, collecting additional information and re-consent. Prior to participation, participants were informed about the objectives and extra procedures of this additional study, for which they provided written informed consent.

\section{Results}

Recruitment took place between October 2016 and February 2017. Out of 454 invited individuals, 356 were recruited (78.4\% participation rate). Participation rate was similar in the T2D (173 out of 227 invited participants) and non-T2D (183 out of 227 invited participants) groups. At baseline, the 356 individuals who accepted to participate in the CHRIS-NAFLD study, were on average 67.6 years old (standard deviation, $\mathrm{SD}=10.6$ ) and 177 (49.7\%) were females. One participant selected in the non-T2D group reported an incident T2D diagnosis at the time of the CHRIS-NAFLD examination and was thus included in the T2D group. We collected saliva and stool samples of 354 and 350 participants, respectively. The DNA extraction and quality control were completed for all samples and they all amplified in PCR. DNA was normalized to $\sim 100 \mathrm{ng} / \mu \mathrm{l}$ and stored at $-80^{\circ} \mathrm{C}$ until further use. 
Table 1 Description of the CHRIS-NAFLD study sample

\begin{tabular}{|c|c|c|}
\hline & \multicolumn{2}{|l|}{ Groups } \\
\hline & $\mathrm{T}^{\mathrm{a}} \mathrm{D}^{\mathrm{a}}(\mathrm{N}=173)$ & Non-T2D ${ }^{a}(N=183)$ \\
\hline Female-n (\%) & $84(48.6)$ & $93(50.8)$ \\
\hline Age (years)—mean (SD) & $67.3(10.4)$ & $67.9(10.7)$ \\
\hline Alcohol consumption (g/day)—median (IQR) & $2.0(0.0,13.5)$ & $4.9(1.0,14.2)$ \\
\hline \multicolumn{3}{|l|}{ Smoking habits—n (\%) } \\
\hline Never & $104(60.5)$ & $114(62.3)$ \\
\hline Past & $55(32.0)$ & $59(32.2)$ \\
\hline Current & $13(7.6)$ & $10(5.5)$ \\
\hline Self-reported diabetes-n (\%) & $111(64.2)$ & $1(0.6)$ \\
\hline Diabetes treatment-n (\%) & $88(51.8)$ & $0(0.0)$ \\
\hline Systolic blood pressure-mean (SD) & $142.1(19.6)$ & $137.6(8.7)$ \\
\hline Anti-hypertensive treatment-n (\%) & $109(63.4)$ & $60(32.8)$ \\
\hline Lipid-lowering agents—n (\%) & $77(45.3)$ & $35(19.9)$ \\
\hline Glycated hemoglobin (\%)—median (IRQ) & $6.4(6.0,6.9)$ & $5.3(5.2,5.4)$ \\
\hline Fasting glucose (mg/dl)—median (IRQ) & $122.5(107.0,142.0)$ & $90.0(85.0,96.0)$ \\
\hline Total cholesterol (mg/dl)—mean (SD) & $199.5(43.7)$ & $215.7(45.9)$ \\
\hline $\mathrm{HDL}(\mathrm{mg} / \mathrm{dl})$-mean (SD) & $53.2(12.1)$ & $58.6(13.6)$ \\
\hline LDL (mg/dl)-mean (SD) & $125.9(40.2)$ & $135.9(40.8)$ \\
\hline Triglycerides (mg/dl)—median (IRQ) & $111.5(87.5,143.5)$ & $91.0(71.0,122.0)$ \\
\hline Proton pump inhibitors-n (\%) & $19(11.2)$ & $17(9.7)$ \\
\hline Statins-n (\%) & $75(44.1)$ & $35(19.9)$ \\
\hline Body-mass-index $\left(\mathrm{kg} / \mathrm{m}^{2}\right)$ —-median (IQR) & $30.0(26.35,32.7)$ & $25.8(23.65,29.0)$ \\
\hline Waist circumference $(\mathrm{cm})$ - mean (SD) & $100.2(13.9)$ & $89.5(12.1)$ \\
\hline Body Fat (\%)—mean (SD) & $35.0(9.6)$ & $31.1(10.1)$ \\
\hline Visceral Fat (\%)—-mean (SD) & $13.2(4.8)$ & $10.5(4.1)$ \\
\hline Metabolic syndrome-n (\%) & $135(78.0)$ & $45(24.6)$ \\
\hline \multicolumn{3}{|l|}{ Hepatic steatosis-n (\%) } \\
\hline Grade 1 & $56(32.4)$ & $115(62.8)$ \\
\hline Grade 2 & $74(42.8)$ & $60(32.8)$ \\
\hline Grade 3 & $43(24.9)$ & $8(4.4)$ \\
\hline Controlled attenuation parameter (dB/m)—median (IQR) & $263(223,315)$ & $234(186,266)$ \\
\hline Liver stiffness (kPa)—median (IQR) & $4.8(3.7,5.9)$ & $3.9(3.3,5.1)$ \\
\hline Visceral adiposity index—median (IQR) & $1.46(1.09,2.16)$ & $1.07(0.77,1.64)$ \\
\hline NAFLD liver fat score - median (IQR) & $0.20(-0.70,1.13)$ & $-1.90(-2.25,-1.24)$ \\
\hline Hepatic steatosis score-median (IQR) & $41.2(36.0,46.0)$ & $34.0(31.6,38.1)$ \\
\hline Fatty liver index-mean (SD) & $41.1(29.6)$ & $55.2(28.6)$ \\
\hline NAFLD fibrosis score - mean (SD) & $-0.55(1.30)$ & $-1.30(1.17)$ \\
\hline Fibrosis (Fib)-4—median (IQR) & $1.22(0.93,1.71)$ & $1.43(1.07,1.80)$ \\
\hline NAFLD classification—n (\%) & $116(63.7)$ & $66(36.3)$ \\
\hline
\end{tabular}

a At CHRIS study baseline

Characteristics of the 356 participants at the time of participation to the CHRIS-NAFLD study are described in Table 1, following the stratification by T2D status that was used for recruitment. Briefly, 173 (48.6\%) and 183 (51.4\%) were T2D and non-T2D, respectively. Mean follow-up time was 3.56 years $(\mathrm{SD}=0.82)$ and 3.91 years (0.88) for T2D and non-T2D participants, respectively.
As expected, T2D and non-T2D participants had similar age and sex distributions. Of the T2D participants, $8.1 \%$ reported to have never consumed alcohol, while $22.5 \%$ drink daily (5.5\% and $29.5 \%$ in the non-T2D group). Most of the participants were never $(60.5 \%$ and $62.3 \%$ for T2D and non-T2D, respectively) or past smokers $(32.0 \%$ and $32.2 \%)$. 
Among T2D individuals, 111 (64.2\%) reported a diagnosis of diabetes, 88 (51.8\%) were on diabetic treatment at the time of the visit, $42.4 \%$ had HbA1c values in the pre-diabetic range (HbA1c 6.0 to $6.4 \%$ ), while $44.2 \%$ had $\mathrm{HbA} 1 \mathrm{c}$ values in the diabetic range $(\mathrm{HbA} 1 \mathrm{c} \geq 6.5 \%)$. Fifty percent of the T2D individuals and $18.3 \%$ of the non$\mathrm{T} 2 \mathrm{D}$ individuals were obese ( $\mathrm{BMI} \geq 30$ ). Hypertension was observed at the time of the visit in $55.9 \%$ of the T2D individuals and $44.2 \%$ of the non-T2D individuals. Use of proton-pump inhibitors (PPIs), which were shown to potentially influence gut microbial composition [73, 74], had similar distribution in T2D and non-T2D participants, while statins were more common in T2D individuals (Table 1 ).

Hepatic steatosis was diagnosed in 43 of the 173 T2D individuals $(24.9 \%)$ and 8 of the 183 non-T2D ones (4.4\%). Furthermore, T2D participants showed higher values of TE than non-T2D participants (median $=4.8$ $(\mathrm{IQR}=3.7,5.9) \mathrm{kPa}$ vs. 3.9 (3.3, 5.1) kPa) (Fig. 1). These findings agreed with non-invasive scoring systems, like NAFLD fibrosis score, suggesting increased liver fibrosis in T2D individuals (Table 1).

According to the criteria for NAFLD calculation, we identified 172 as affected with NAFLD and 181 healthy individuals among the two groups of T2D and not-T2D participants. Power calculations are shown in Fig. 2. Assuming we wish to compare the relative bacterial abundance in participants affected with NAFLD versus healthy ones and analyzing data using a Fisher exact test, we anticipate a $>80 \%$ power to detect a difference in the proportion of relative abundance of single bacterial strains in the two groups higher than 15\% (Fig. 2, scenario 1 ). Despite previous studies that did not identify more than 6 phyla, we predict finding 10 phyla (Fig. 2, scenario 2 ); in this scenario, the study will have $80 \%$ power to detect differences in the proportions equal or higher than 20\%. In Fig. 2, scenario 3, we consider 250 bacterial subgroups, calculated assuming to find 10 phyla, 5 families per phyla and 5 genera per family. Under this scenario the study would have $80 \%$ power to detect differences in proportions higher than $22.5 \%$ in most cases. However, in terms of multiple comparisons, this should be considered as a pessimistic scenario as none of previous studies was able to identify phyla, families and genera at such a high level of detail.

\section{Discussion}

CHRIS-NAFLD is a population-based study aimed to assess the relationship between gut and oral microbiota and NAFLD in individuals with and without T2D. Stratification by diabetes will enable assessment of whether the NAFLD-microbiota relationship is modified or moderated by, or is independent of, the T2D status. In addition to the cross-sectional nature of the investigation, the

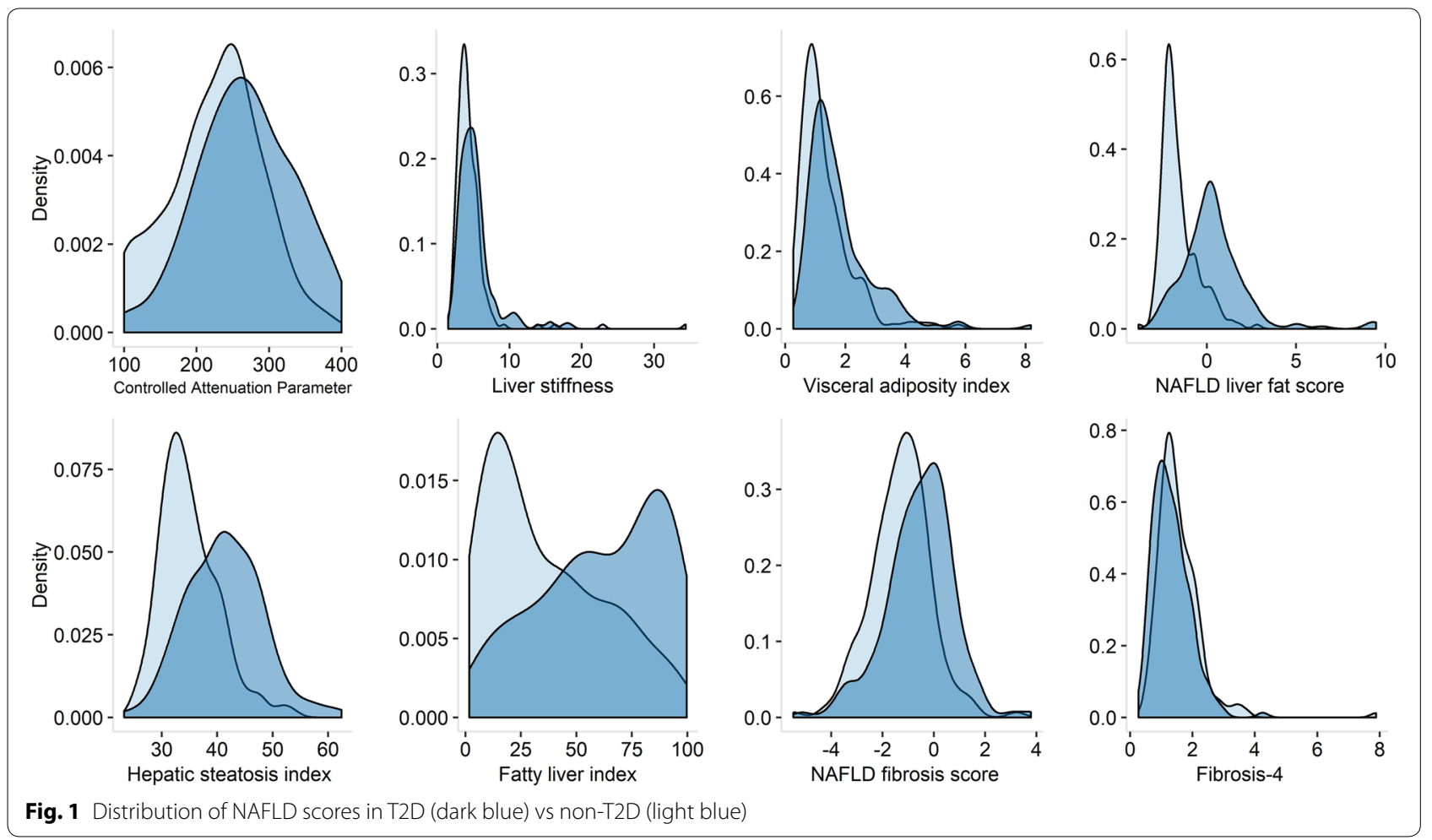



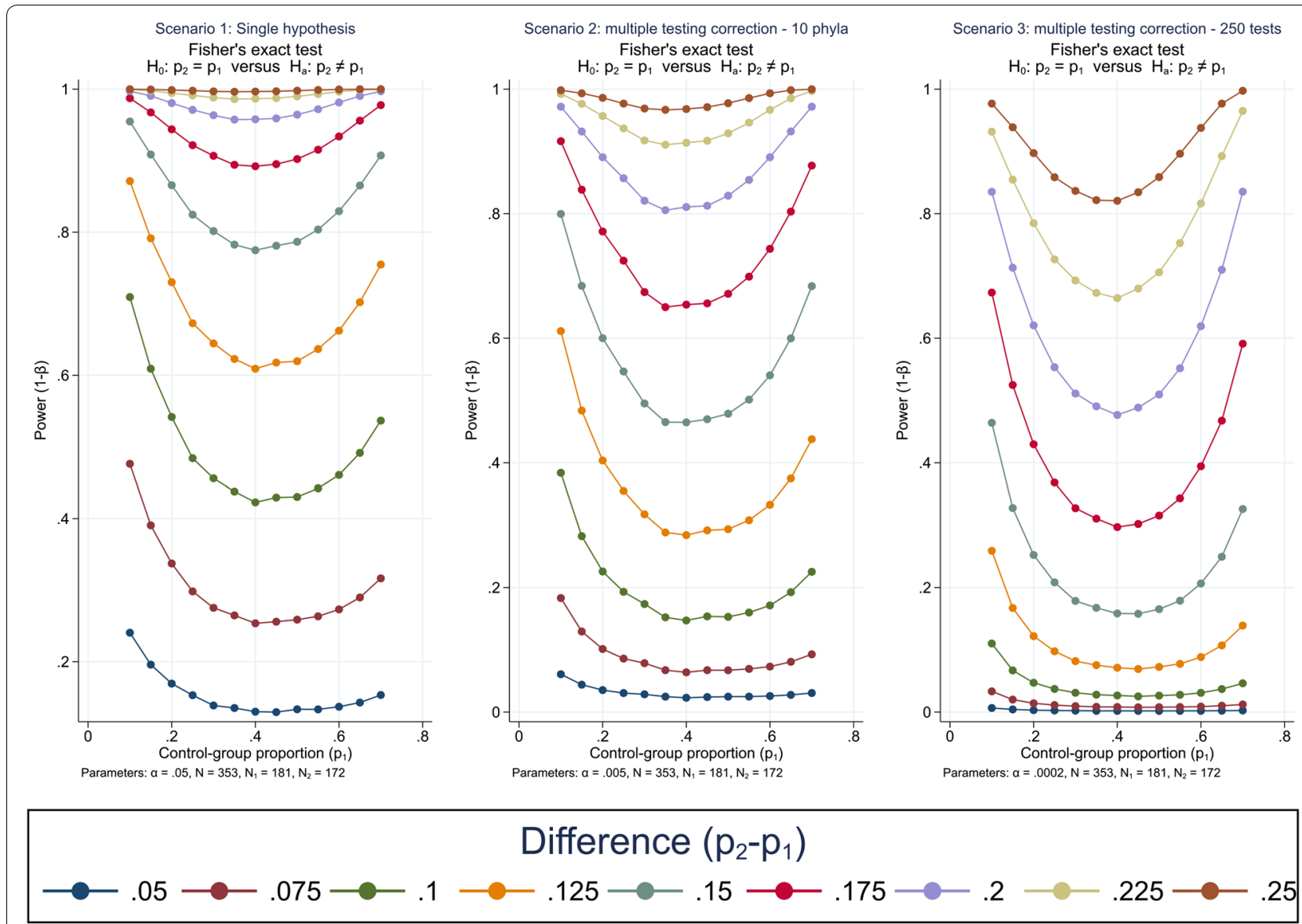

Fig. 2 Power to detect given differences between bacterial abundance in 172 NAFLD affected versus 181 non-affected individuals under three different scenarios: a single hypothesis (significance level, $a=0.05$ ), correction for multiple testing of 10 phyla (Bonferroni-corrected $a=0.005$ ), and correction for 250 independent tests $(a=0.0002) . p_{1}=$ proportion of bacteria in non-NAFLD individuals; $p_{2}=$ proportion of bacteria in NAFLD individuals

study will help to evaluate whether NAFLD assessed at the time of the CHRIS-NAFLD participation was associated with clinical or biochemical information collected at the time of the CHRIS baseline participation. Finally, the study will provide important information on the relationship between microbiota measured at different sites of the human body, specifically from samples of saliva and stool.

Currently, extensive efforts are being focused on the analyses of microbiota in relation to several diseases. In recent years, the importance of gut microbiota in NAFLD was demonstrated by several independent groups. NAFLD patients exhibit distinct changes in their intestinal flora, which impact the host metabolism [75]. Patients show a higher abundance of bacterial strains which supply the host with nutrient source out of indigestible products, such as complex carbohydrates [21, 76]. However, studies conducted in the general population on the association between microbiota and NAFLD are few and with a smaller number of participants compared to ours [32, 77-79].

It is likely that the interaction of genetic and environmental factors with metabolic alterations accelerate NAFLD progression in T2D patients [80]. NAFLD and T2D commonly co-exist and several studies have demonstrated that NAFLD might be found in up to $70 \%$ of patients with T2D $[6,81]$. From this perspective, our study will allow the question as to whether the NAFLDmicrobiota relationship is affected by the diabetic status to be answered.

In a landmark work, Qin and colleagues defined a distinct microbial composition in late stage liver disease. In liver cirrhosis patients, the specific beneficial bacterial strains, like Faecalibacterium prausnitzii, were diminished compared to healthy individuals. Additional analyses of buccal flora showed that, in the patients with liver cirrhosis, harmful bacterial strains are transferred from the oral cavity to the intestine, possibly contributing to 
the development of cirrhosis [43]. In terms of "oralisation" of the intestinal microbiota, the widespread use of PPIs, which reduce the barrier function of gastric acid [74], received great attention in the recent years. Several studies analyzed the impact of PPIs on liver diseases such as hepatic encephalopathy [82] and alcoholic liver disease [83]. Therefore, there is an urgent need to understand the pathophysiological mechanisms leading to NAFLD and to gain more insight into the role of intestinal and oral microbiota in NAFLD.

In the CHRIS-NAFLD study, we collected both saliva and faeces for microbiota analyses. The oral and gastrointestinal microbiome represents the bulk of the overall human microbial load. The correlation of oral microbiota and gut microbiota in NAFLD patients has not been evaluated yet. This will give novel insights into the composition of the microbiota in individuals with and without T2D, possibly identifying microbial transfer in NAFLD patients. Moreover, since saliva is easier to collect compared to stool, if we will observe a similar composition of salivary and stool microbiota, this would increase the compliance of individual participation in such microbiota studies.

In a preliminary descriptive analysis we observed a higher level of liver stiffness in T2D participants, a finding also reported in other cohorts [84]. We also observed a higher prevalence of NAFLD-affected individuals in the T2D groups, as reported by other studies $[6,7]$.

Strengths of this study include comprehensive assessment of NAFLD, T2D and microbiota in individuals from the general population submitted to a comprehensive evaluation of their hepatic health via ultrasound and elastography examination. In addition to the described data, a wealth of additional genetic, molecular, clinical, environmental data and biological biobanked samples collected in the framework of the CHRIS study are available $[44,45,85]$. Our study has also potential limitations. Even though liver biopsy represents the gold standard for the diagnosis of fibrosis, we used TE for this assessment since the use of an invasive procedure, such as biopsy, would not be ethically justifiable in a population-based observational study. Furthermore, TE is considered a valid noninvasive alternative for this assessment, as reported previously [86]. The ultrasound based method used for detection of steatosis has $85 \%$ sensitivity and $94 \%$ specificity for identifying a degree of $\geq 20-30 \%$ steatosis [87]. Finally, we classified participants that had fasting HbAlc levels of $\geq 6.5 \%$ as having T2D since the recent International Expert Committee's statements recommended using these HbA1c levels as diagnostic criteria for diabetes [46], but there are also some potential factors that can lead to altered HbA1c levels such as chronic salicylate intake in some individuals [88]. Furthermore, the daily medication of participants was also evaluated in our study. PPIs were equally distributed in T2D and non-T2D individuals. In a large meta-analysis, PPIs were shown to be associated with increased enteric infection with Clostridium difficile [89]. Statins, which were also proven to influence gut microbiota in mice [90,91], were more commonly reported by T2D individuals. This difference could be explained by higher numbers of dyslipidemia in T2D participants.

In summary, by combining comprehensive bio-sampling with clinical characterization including detailed information on drug history of a large group of individuals with or without T2D and related NAFLD, the CHRISNAFLD study will help elucidate important questions on the relationship between microbiota and presence of NAFLD in patients with and without T2D.

\section{Abbreviations}

ALT: alanine transaminase; AST: aspartate transaminase; ATC: anatomical therapeutic chemical; BMI: body mass index; BP: blood pressure; BSS: Bristol stool scale; CHRIS: Cooperative Health Research In South Tyrol; DM: diabetes mellitus; FIB-4: fibrosis-4; FFQ: Food Frequency Questionnaire; FLI: fatty liver index; FPG: fasting plasma glucose; GGT: gamma-glutamyl transferase; GWAS: genome-wide association study; HbA1c: glycated hemoglobin; HDL: high-density lipoproteins; HIS: hepatic steatosis score; HOMA-IR: homeostatic model assessment-insulin resistance; IFG: impaired fasting glucose; IQR: interquartile range; LFS: NAFLD liver fat score; MetS: metabolic syndrome; NAFLD: non-alcoholic fatty liver disease; NASH: non-alcoholic steatohepatitis; NFS: NAFLD fibrosis score; OHQ: Oral Health Questionnaire; PLT: platelets; PPIs: proton-pump inhibitors; RDP: ribosomal database project; SCFAs: short chain fatty acids; SD: standard deviation; T2D: type 2 diabetes; TE: transient elastography; TG: triglyceride; VAl: visceral adiposity index; WC: waist circumference.

\section{Acknowledgements}

We thank all study participants, the study personnel, and Dr. Helmuth Weiss (Hospital of Silandro) for his collaboration and supervision; the Biobank's laboratory technicians and Dr. Claudia Volpato of the Institute for Biomedicine for her collaboration in libraries' preparation; the IT support and data manager of the Institute for Biomedicine; Dr. Andrew Hicks of the Institute for Biomedicine for the linguistic revision. We thank the Labormed Srl - GmbH, Bolzano (represented by Dr. Monica Cembran) for funding the insulin measurements, which were performed by Synlab Italia Srl, Castenedolo. The authors thank the Department of Innovation, research and University of autonomous Province of Bozen/Bolzano for covering the Open Access publication costs.

\section{Authors' contributions}

BMM, CG, MG, LF, W, RM, ADG, AP, DM, AR, CP, HT and PPP drafted the manuscript. HT and PPP planned and designed the study; BMM, CG, MG, LF, VV, RM, $C F, A D G, C C, A P, D M, A R, C P, H T$ and PPP critically reviewed the manuscript. All authors read and approved the final manuscript.

\section{Funding}

This study was supported by the Department of Innovation and Research and University of the Autonomous Province of Bolzano.

\section{Availability of data and materials}

The datasets generated and/or analyzed during the current study are available from the corresponding author on reasonable request.

\section{Ethics approval and consent to participate}

The CHRIS-NAFLD study protocol was approved by the Ethical Committee of the Healthcare System of the Autonomous Province of Bolzano (Südtiroler Sanitätsbetrieb/Azienda Sanitaria dell'Alto Adige), protocol no. 85-2016 (19 
Oct 2016). Participants were informed on the objectives of this study and provided written informed consent.

\section{Consent for publication}

Not applicable.

\section{Competing interests}

The authors declare that they have no competing interests.

\section{Author details}

${ }^{1}$ Institute for Biomedicine (affiliated to the University of Lübeck), Eurac Research, 39100 Bolzano, Italy. ${ }^{2}$ Department of Internal Medicine I, Gastroenterology, Hepatology, Endocrinology \& Metabolism, Medical University Innsbruck, Innsbruck, Austria. ${ }^{3}$ Center for Research Ethics and Bioethics, Department of Public Health and Caring Science, Uppsala University, Uppsala, Sweden.

Received: 26 June 2019 Accepted: 7 November 2019 Published online: 04 December 2019

\section{References}

1. Vernon G, Baranova A, Younossi ZM. Systematic review: the epidemiology and natural history of non-alcoholic fatty liver disease and non-alcoholic steatohepatitis in adults. Aliment Pharmacol Ther. 2011;34(3):274-85.

2. Singh S, Allen AM, Wang Z, Prokop LJ, Murad MH, Loomba R. Fibrosis progression in nonalcoholic fatty liver vs nonalcoholic steatohepatitis: a systematic review and meta-analysis of paired-biopsy studies. Clin Gastroenterol Hepatol. 2015;13(4):643-54.e1-9 (quiz e39-40).

3. Younossi ZM, Koenig AB, Abdelatif D, Fazel Y, Henry L, Wymer M. Global epidemiology of nonalcoholic fatty liver disease-meta-analytic assessment of prevalence, incidence, and outcomes. Hepatology. 2016;64(1):73-84.

4. Charlton MR, Burns JM, Pedersen RA, Watt KD, Heimbach JK, Dierkhising RA. Frequency and outcomes of liver transplantation for nonalcoholic steatohepatitis in the United States. Gastroenterology. 2011;141(4):1249-53.

5. Bellentani S, Saccoccio G, Masutti F, Croce LS, Brandi G, Sasso F, et al. Prevalence of and risk factors for hepatic steatosis in Northern Italy. Ann Intern Med. 2000;132(2):112-7.

6. Targher G, Bertolini L, Padovani R, Rodella S, Tessari R, Zenari L, et al. Prevalence of nonalcoholic fatty liver disease and its association with cardiovascular disease among type 2 diabetic patients. Diabetes Care. 2007;30(5):1212-8.

7. Leite NC, Salles GF, Araujo AL, Villela-Nogueira CA, Cardoso CR. Prevalence and associated factors of non-alcoholic fatty liver disease in patients with type-2 diabetes mellitus. Liver Int. 2009;29(1):113-9.

8. Adams LA, Waters OR, Knuiman MW, Elliott RR, Olynyk JK. NAFLD as a risk factor for the development of diabetes and the metabolic syndrome: an eleven-year follow-up study. Am J Gastroenterol. 2009;104(4):861-7.

9. Zelber-Sagi S, Lotan R, Shibolet O, Webb M, Buch A, Nitzan-Kaluski D, et al. Non-alcoholic fatty liver disease independently predicts prediabetes during a 7-year prospective follow-up. Liver Int. 2013;33(9):1406-12.

10. Neuschwander-Tetri BA, Caldwell SH. Nonalcoholic steatohepatitis: summary of an AASLD Single Topic Conference. Hepatology. 2003;37(5):1202-19.

11. Alisi A, Ceccarelli S, Panera N, Nobili V. Causative role of gut microbiota in non-alcoholic fatty liver disease pathogenesis. Front Cell Infect Microbiol. 2012;2:132.

12. De Gottardi A, McCoy KD. Evaluation of the gut barrier to intestinal bacteria in non-alcoholic fatty liver disease. J Hepatol. 2011;55(6):1181-3.

13. Csak T, Velayudham A, Hritz I, Petrasek J, Levin I, Lippai D, et al. Deficiency in myeloid differentiation factor-2 and toll-like receptor 4 expression attenuates nonalcoholic steatohepatitis and fibrosis in mice. Am J Physiol Gastrointest Liver Physiol. 2011;300(3):G433-41.

14. Wang BH, Yao MF, LV LX, Ling ZX, Li LJ. The human microbiota in health and disease. Eng Prc. 2017;3(1):71-82.

15. Langdon A, Crook N, Dantas G. The effects of antibiotics on the microbiome throughout development and alternative approaches for therapeutic modulation. Genome Med. 2016;8(1):39.
16. Karlsson F, Tremaroli V, Nielsen J, Backhed F. Assessing the human gut microbiota in metabolic diseases. Diabetes. 2013;62(10):3341-9.

17. Khan MT, Nieuwdorp M, Backhed F. Microbial modulation of insulin sensitivity. Cell Metab. 2014;20(5):753-60.

18. Qin J, Li Y, Cai Z, Li S, Zhu J, Zhang F, et al. A metagenome-wide association study of gut microbiota in type 2 diabetes. Nature. 2012;490(7418):55-60.

19. Le Chatelier E, Nielsen T, Qin J, Prifti E, Hildebrand F, Falony G, et al. Richness of human gut microbiome correlates with metabolic markers. Nature. 2013;500(7464):541-6.

20. Muscogiuri G, Balercia G, Barrea L, Cignarelli A, Giorgino F, Holst JJ, et al. Gut: a key player in the pathogenesis of type 2 diabetes? Crit Rev Food Sci Nutr. 2018:58(8):1294-309.

21. Turnbaugh PJ, Ley RE, Mahowald MA, Magrini V, Mardis ER, Gordon J. An obesity-associated gut microbiome with increased capacity for energy harvest. Nature. 2006:444(7122):1027-31.

22. Ley RE, Turnbaugh PJ, Klein S, Gordon Jl. Microbial ecology: human gut microbes associated with obesity. Nature. 2006;444(7122):1022-3.

23. Million M, Maraninchi M, Henry M, Armougom F, Richet H, Carrieri P, et al. Obesity-associated gut microbiota is enriched in Lactobacillus reuteri and depleted in Bifidobacterium animalis and Methanobrevibacter smithii. Int J Obes (Lond). 2012;36(6):817-25.

24. Louis P, Scott KP, Duncan SH, Flint HJ. Understanding the effects of diet on bacterial metabolism in the large intestine. J Appl Microbiol. 2007;102(5):1197-208.

25. Barrea L, Muscogiuri G, Annunziata G, Laudisio D, Pugliese G, Salzano C, et al. From gut microbiota dysfunction to obesity: could short-chain fatty acids stop this dangerous course? Hormones (Athens). 2019;18:245-50.

26. Schwiertz A, Taras D, Schafer K, Beijer S, Bos NA, Donus C, et al. Microbiota and SCFA in lean and overweight healthy subjects. Obesity (Silver Spring). 2010;18(1):190-5.

27. Sanna S, van Zuydam NR, Mahajan A, Kurilshikov A, Vich Vila A, Vosa U, et al. Causal relationships among the gut microbiome, short-chain fatty acids and metabolic diseases. Nat Genet. 2019;51(4):600-5.

28. Raman M, Ahmed I, Gillevet PM, Probert CS, Ratcliffe NM, Smith S, et al. Fecal microbiome and volatile organic compound metabolome in obese humans with nonalcoholic fatty liver disease. Clin Gastroenterol Hepatol. 2013;11(7):868-75.e1-3.

29. Mouzaki M, Comelli EM, Arendt BM, Bonengel J, Fung SK, Fischer SE, et al. Intestinal microbiota in patients with nonalcoholic fatty liver disease. Hepatology. 2013;58(1):120-7.

30. Zhang X, Shen D, Fang Z, Jie Z, Qiu X, Zhang C, et al. Human gut microbiota changes reveal the progression of glucose intolerance. PLOS ONE. 2013;8(8):e71108.

31. Wong VW, Tse CH, Lam TT, Wong GL, Chim AM, Chu WC, et al. Molecular characterization of the fecal microbiota in patients with nonalcoholic steatohepatitis - a longitudinal study. PLoS ONE. 2013;8(4):e62885.

32. Boursier J, Mueller O, Barret M, Machado M, Fizanne L, Araujo-Perez F, et al. The severity of nonalcoholic fatty liver disease is associated with gut dysbiosis and shift in the metabolic function of the gut microbiota. Hepatology. 2016;63(3):764-75.

33. Del Chierico F, Nobili V, Vernocchi P, Russo A, Stefanis C, Gnani D, et al. Gut microbiota profiling of pediatric nonalcoholic fatty liver disease and obese patients unveiled by an integrated meta-omics-based approach. Hepatology. 2017;65(2):451-64.

34. Loomba R, Seguritan V, Li W, Long T, Klitgord N, Bhatt A, et al. Gut microbiome-based metagenomic signature for non-invasive detection of advanced fibrosis in human nonalcoholic fatty liver disease. Cell Metab. 2017;25(5):1054-62.e5.

35. Dewhirst FE, Chen T, Izard J, Paster BJ, Tanner AC, Yu WH, et al. The human oral microbiome. J Bacteriol. 2010;192(19):5002-17.

36. Belda-Ferre P, Alcaraz LD, Cabrera-Rubio R, Romero H, Simon-Soro A, Pignatelli $M$, et al. The oral metagenome in health and disease. ISME J. 2012:6(1):46-56.

37. Huang S, Yang F, Zeng X, Chen J, Li R, Wen T, et al. Preliminary characterization of the oral microbiota of Chinese adults with and without gingivitis. BMC Oral Health. 2011;11:33

38. Casarin RC, Barbagallo A, Meulman T, Santos VR, Sallum EA, Nociti FH, et al. Subgingival biodiversity in subjects with uncontrolled type-2 diabetes and chronic periodontitis. J Periodontal Res. 2013;48(1):30-6. 
39. Hintao J, Teanpaisan R, Chongsuvivatwong V, Ratarasan C, Dahlen G. The microbiological profiles of saliva, supragingival and subgingival plaque and dental caries in adults with and without type 2 diabetes mellitus. Oral Microbiol Immunol. 2007;22(3):175-81.

40. Alazawi W, Bernabe E, Tai D, Janicki T, Kemos P, Samsuddin S, et al. Periodontitis is associated with significant hepatic fibrosis in patients with non-alcoholic fatty liver disease. PLoS ONE. 2017;12(12):e0185902.

41. Yoneda M, Naka S, Nakano K, Wada K, Endo H, Mawatari H, et al. Involvement of a periodontal pathogen, Porphyromonas gingivalis on the pathogenesis of non-alcoholic fatty liver disease. BMC Gastroenterol. 2012;12:16.

42. Acharya C, Sahingur SE, Bajaj JS. Microbiota, cirrhosis, and the emerging oral-gut-liver axis. JCI Insight. 2017;2(19):e94416.

43. Qin N, Yang F, Li A, Prifti E, Chen Y, Shao L, et al. Alterations of the human gut microbiome in liver cirrhosis. Nature. 2014;513(7516):59-64.

44. Pattaro C, Gogele M, Mascalzoni D, Melotti R, Schwienbacher C, De Grandi A, et al. The Cooperative Health Research in South Tyrol (CHRIS) study: rationale, objectives, and preliminary results. J Transl Med. 2015;13:348.

45. Noce D, Gogele M, Schwienbacher C, Caprioli G, De Grandi A, Foco L, et al. Sequential recruitment of study participants may inflate genetic heritability estimates. Hum Genet. 2017;136(6):743-57.

46. Standards of Medical Care in Diabetes. American Diabetes Association. Diabetes care. 2014;37(Suppl 1):S14-80. https://doi.org/10.2337/ dc14-S014

47. Waist circumference and waist-hip ratio. Report of a WHO expert consultation, Geneva, 8-11 December 2008.

48. Garcia-Larsen V, Luczynska M, Kowalski ML, Voutilainen H, Ahlstrom M, Haahtela T, et al. Use of a common food frequency questionnaire (FFQ) to assess dietary patterns and their relation to allergy and asthma in Europe: pilot study of the GA2LEN FFQ. Eur J Clin Nutr. 2011;65(6):750-6.

49. Chinn S, Jarvis D, Melotti R, Luczynska C, Ackermann-Liebrich U, Anto JM, et al. Smoking cessation, lung function, and weight gain: a follow-up study. Lancet. 2005;365(9471):1629-35 (discussion 00-1)

50. Castera L. Assessing liver fibrosis. Expert Rev Gastroenterol Hepatol. 2008:2(4):541-52.

51. Vuppalanchi R, Siddiqui MS, Van Natta ML, Hallinan E, Brandman D, Kowdley K, et al. Performance characteristics of vibration-controlled transient elastography for evaluation of nonalcoholic fatty liver disease. Hepatology. 2018;67(1):134-44.

52. Wong VW, Vergniol J, Wong GL, Foucher J, Chan HL, Le Bail B, et al. Diagnosis of fibrosis and cirrhosis using liver stiffness measurement in nonalcoholic fatty liver disease. Hepatology. 2010;51(2):454-62.

53. Siddiqui MS, Vuppalanchi R, Van Natta ML, Hallinan E, Kowdley KV, Abdelmalek $M$, et al. Vibration-controlled transient elastography to assess fibrosis and steatosis in patients with nonalcoholic fatty liver disease. Clin Gastroenterol Hepatol. 2019;17(1):156-63 e2.

54. Ballestri S, Lonardo A, Romagnoli D, Carulli L, Losi L, Day CP, et al. Ultrasonographic fatty liver indicator, a novel score which rules out NASH and is correlated with metabolic parameters in NAFLD. Liver Int. 2012;32(8):1242-52.

55. Sturm W, Sandhofer A, Engl J, Laimer M, Molnar C, Kaser S, et al. Influence of visceral obesity and liver fat on vascular structure and function in obese subjects. Obesity (Silver Spring). 2009;17(9):1783-8.

56. Abd El-Kader SM, El-Den Ashmawy EM. Non-alcoholic fatty liver disease: the diagnosis and management. World J Hepatol. 2015;7(6):846-58.

57. IDF Consensus Worldwide Definition of the Metabolic Syndrome.

58. Zimmet P, Magliano D, Matsuzawa Y, Alberti G, Shaw J. The metabolic syndrome: a global public health problem and a new definition. J Atheroscler Thromb. 2005;12(6):295-300.

59. Amato MC, Giordano C, Galia M, Criscimanna A, Vitabile S, Midiri M, et al. Visceral Adiposity Index: a reliable indicator of visceral fat function associated with cardiometabolic risk. Diabetes Care. 2010;33(4):920-2.

60. Kotronen A, Peltonen M, Hakkarainen A, Sevastianova K, Bergholm $R$, Johansson $L M$, et al. Prediction of non-alcoholic fatty liver disease and liver fat using metabolic and genetic factors. Gastroenterology. 2009;137(3):865-72.

61. Lee JH, Kim D, Kim HJ, Lee CH, Yang Jl, Kim W, et al. Hepatic steatosis index: a simple screening tool reflecting nonalcoholic fatty liver disease. Dig Liver Dis. 2010;42(7):503-8. https://doi.org/10.1016/j.dld.2009.08.002.
62. Bedogni G, Bellentani S, Miglioli L, Masutti F, Passalacqua M, Castiglione A, et al. The Fatty Liver Index: a simple and accurate predictor of hepatic steatosis in the general population. BMC Gastroenterol. 2006;6:33.

63. Angulo P, Hui JM, Marchesini G, Bugianesi E, George J, Farrell GC, et al. The NAFLD fibrosis score: a noninvasive system that identifies liver fibrosis in patients with NAFLD. Hepatology. 2007;45:846-54. https://doi. org/10.1002/hep.21496

64. Sterling RK, Lissen E, Clumeck N, Sola R, Correa MC, Montaner J, et al. Development of a simple noninvasive index to predict significant fibrosis in patients with HIV/HCV coinfection. Hepatology. 2006;43(6):1317-25.

65. Matthews DR, Hosker JP, Rudenski AS, Naylor BA, Treacher DF, Turner RC. Homeostasis model assessment: insulin resistance and beta-cell function from fasting plasma glucose and insulin concentrations in man. Diabetologia. 1985;28(7):412-9.

66. Mabile L, Dalgleish R, Thorisson GA, Deschenes M, Hewitt R, Carpenter J, et al. Quantifying the use of bioresources for promoting their sharing in scientific research. Gigascience. 2013;2(1):7.

67. O'Donnell LJ, Virjee J, Heaton KW. Detection of pseudodiarrhoea by simple clinical assessment of intestinal transit rate. BMJ. 1990;300(6722):439-40.

68. Chumpitazi BP, Self MM, Czyzewski DI, Cejka S, Swank PR, Shulman RJ. Bristol Stool Form Scale reliability and agreement decreases when determining Rome III stool form designations. Neurogastroenterol Motil. 2016:28(3):443-8.

69. Riegler G, Esposito I. Bristol scale stool form. A still valid help in medical practice and clinical research. Tech Coloproctol. 2001;5(3):163-4.

70. World Health Organization (WHO). Oral health surveys: basic methods, 5th edition. WHO

71. Wang J, Kurilshikov A, Radjabzadeh D, Turpin W, Croitoru K, Bonder MJ, et al. Meta-analysis of human genome-microbiome association studies: the MiBioGen consortium initiative. Microbiome. 2018:6(1):101.

72. Michigan Imputation Server. https://imputationserver.sph.umich.edu/ index.html\#!

73. Imhann F, Bonder MJ, Vich Vila A, Fu J, Mujagic Z, Vork L, et al. Proton pump inhibitors affect the gut microbiome. Gut. 2016:65(5):740-8.

74. Jackson MA, Goodrich JK, Maxan ME, Freedberg DE, Abrams JA, Poole AC, et al. Proton pump inhibitors alter the composition of the gut microbiota. Gut. 2016:65(5):749-56.

75. Abdul-Hai A, Abdallah A, Malnick SD. Influence of gut bacteria on development and progression of non-alcoholic fatty liver disease. World $J$ Hepatol. 2015;7(12):1679-84.

76. Samuel BS, Gordon II. A humanized gnotobiotic mouse model of host-archaeal-bacterial mutualism. Proc Natl Acad Sci USA. 2006;103(26):10011-6.

77. Lelouvier B, Servant F, Paisse S, Brunet AC, Benyahya S, Serino M, et al. Changes in blood microbiota profiles associated with liver fibrosis in obese patients: a pilot analysis. Hepatology. 2016:64(6):2015-27.

78. Spencer MD, Hamp TJ, Reid RW, Fischer LM, Zeisel SH, Fodor AA. Association between composition of the human gastrointestinal microbiome and development of fatty liver with choline deficiency. Gastroenterology. 2011;140(3):976-86.

79. Yun Y, Kim HN, Lee EJ, Ryu S, Chang Y, Shin H, et al. Fecal and blood microbiota profiles and presence of nonalcoholic fatty liver disease in obese versus lean subjects. PLOS ONE. 2019;14(3):e0213692.

80. Leite NC, Villela-Nogueira CA, Cardoso CR, Salles GF. Non-alcoholic fatty liver disease and diabetes: from physiopathological interplay to diagnosis and treatment. World J Gastroenterol. 2014;20(26):8377-92.

81. Williamson RM, Price JF, Glancy S, Perry E, Nee LD, Hayes PC, et al. Prevalence of and risk factors for hepatic steatosis and nonalcoholic Fatty liver disease in people with type 2 diabetes: the Edinburgh Type 2 Diabetes Study. Diabetes Care. 2011;34(5):1139-44.

82. Bian J, Wang A, Lin J, Wu L, Huang H, Wang S, et al. Association between proton pump inhibitors and hepatic encephalopathy: a meta-analysis. Medicine (Baltimore). 2017:96(17):e6723.

83. Llorente $C$, Jepsen $P$, Inamine $T$, et al. Gastric acid suppression promotes alcoholic liver disease by inducing overgrowth of intestinal Enterococcus. Nat Commun. 2017;8(1):837. https://doi.org/10.1038/s41467-017-00796-X

84. Koehler EM, Plompen EP, Schouten JN, Hansen BE, Darwish Murad S, Taimr $P$, et al. Presence of diabetes mellitus and steatosis is associated with liver stiffness in a general population: the Rotterdam study. Hepatology. 2016;63(1):138-47. 
85. Melotti R, Ruscheweyh R, Pramstaller PP, Hicks AA, Pattaro C. Structural Consistency of the Pain Sensitivity Questionnaire in the Cooperative Health Research In South Tyrol (CHRIS) Population-Based Study. J Pain. 2018;19(12):1424-34.

86. Dhyani M, Anvari A, Samir AE. Ultrasound elastography: liver. Abdom Imaging. 2015;40(4):698-708.

87. Hernaez R, Lazo M, Bonekamp S, Kamel I, Brancati FL, Guallar E, et al. Diagnostic accuracy and reliability of ultrasonography for the detection of fatty liver: a meta-analysis. Hepatology. 2011;54(3):1082-90.

88. Radin MS. Pitfalls in hemoglobin A1c measurement: when results may be misleading. J Gen Intern Med. 2014;29(2):388-94.
89. Janarthanan S, Ditah I, Adler DG, Ehrinpreis MN. Clostridium difficileassociated diarrhea and proton pump inhibitor therapy: a meta-analysis. Am J Gastroenterol. 2012;107(7):1001-10.

90. Caparros-Martin JA, Lareu RR, Ramsay JP, Peplies J, Reen FJ, Headlam HA, et al. Statin therapy causes gut dysbiosis in mice through a PXR-dependent mechanism. Microbiome. 2017;5(1):95.

91. Khan TJ, Ahmed YM, Zamzami MA, Mohamed SA, Khan I, Baothman OAS, et al. Effect of atorvastatin on the gut microbiota of high fat diet-induced hypercholesterolemic rats. Sci Rep. 2018;8(1):662.

\section{Publisher's Note}

Springer Nature remains neutral with regard to jurisdictional claims in published maps and institutional affiliations.
Ready to submit your research? Choose BMC and benefit from:

- fast, convenient online submission

- thorough peer review by experienced researchers in your field

- rapid publication on acceptance

- support for research data, including large and complex data types

- gold Open Access which fosters wider collaboration and increased citations

- maximum visibility for your research: over $100 \mathrm{M}$ website views per year

At BMC, research is always in progress.

Learn more biomedcentral.com/submissions 